\title{
Strongyloides stercoralis: ultrastructural study of newly hatched larvae within human duodenal
} mucosa

Daniele Dionisio, Lidia Ibba Manneschi, Simonetta di Lollo, Alessandra Orsi, Alessia

Tani, Alessandra Papucci, Francesco Esperti, Francesco Leoncini

Infectious Diseases Unit, Pistoia Hospital, Piazza Giovanni XXIII, 51100 Pistoia, Italy

D Dionisio

F Esperti

Department of Human Anatomy and Histology, University of Florence, Florence, Italy

L Ibba Manneschi

A Tani

Institute of Pathology, University of Florence S di Lollo

A Papucci

Laboratory for Microbiology and Virology, Careggi Hospital, Florence, Italy

A Orsi

Infectious Diseases Unit, Careggi Hospital F Leoncini

Correspondence to: Dr Dionisio email:

infdispt@1ycosmail.com

Accepted for publication 17 August 1999

\begin{abstract}
Aim-To investigate the ultrastructural features of the newly hatched larvae of Strongyloides stercoralis in human duodenal mucosa.
\end{abstract}

Methods-Duodenal biopsies from an AIDS patient were studied by transmission electron microscopy to investigate morphology, location, and host-worm relations of newly hatched larvae.

Results-Newly hatched larvae were found in the Lieberkuhn crypts within the tunnels formed by migration of parthenogenic females. Delimiting enterocytes were compressed. Release of larvae into the gut lumen was also documented. It was shown that both a thin and a thick membrane surrounded the eggs and larvae, as a tegument derived respectively from parasite and host. Segmentary spike-like waves, caused by contractures of worm body musculature, were observed on the surface of newly hatched larvae, and their intestinal lumen was closed and empty, with no budding microvilli. Immaturity of the cuticle and some degree immaturity of amphidial neurones were found, but there was no evidence of either immaturity or signs of damage to other structures.

Conclusions-Newly hatched larvae of $S$ stercoralis appear to be a non-feeding immature stage capable of active movement through the epithelium, causing mechanical damage. The tegument resulting from the thin and the thick membrane may protect the parasite and reduce any disadvantage caused by immaturity.

(f Clin Pathol 2000;53:110-116)

Keywords: Strongyloides stercoralis; ultrastructure; larvae; immune deficiency

Strongyloides stercoralis is a worldwide parasitic nematode which is endemic in many developing countries. ${ }^{12}$ Infection occurs when free living third stage larvae penetrate the skin, pass through the circulation to the lungs, enter the airways, and are then swallowed. In the small intestinal mucosa, third stage larvae develop into parthenogenic females which deposit eggs which then release first stage larvae. ${ }^{2}$

Despite its central role in the establishment of parasitism, some aspects of the parasitic life cycle within the small intestinal mucosa of the human, such as the ultrastructural morphology of developing stages, their location, and the host-worm relations, including inflammatory reactions, are not yet fully understood.

To date, no ultrastructural studies on this parasite have been reported in the human, although there have been electron microscopical studies on free living filariform larvae and on adult worms in gut biopsies from immunosuppressed dogs. ${ }^{3-6}$ These studies are useful in providing information on the ultrastructural features, location, and host-worm relations of stages that also parasitise humans, but it is uncertain whether the results can be simply transferred to the human disease. Furthermore, to our knowledge none of these studies has provided any information about ultrastructure and host-worm relations of the earliest stages found within the small intestinal mucosa, that is, the newly hatched larvae.

This study was undertaken to investigate the ultrastructural morphology, location, and hostworm relations of the newly hatched larvae of $S$ stercoralis in duodenal mucosa of humans by comparison with reported electron microscopical features of both free living filariform larvae and adult stages in parasitised animals, and also by considering species other than $S$ stercoralis. ${ }^{3-12}$

Ultrastructural information arising from this study would be expected to provide some insight into the roles played by newly hatched larvae in the human small intestinal mucosa, including host recognition, migration, feeding, and damage to the intestinal cells.

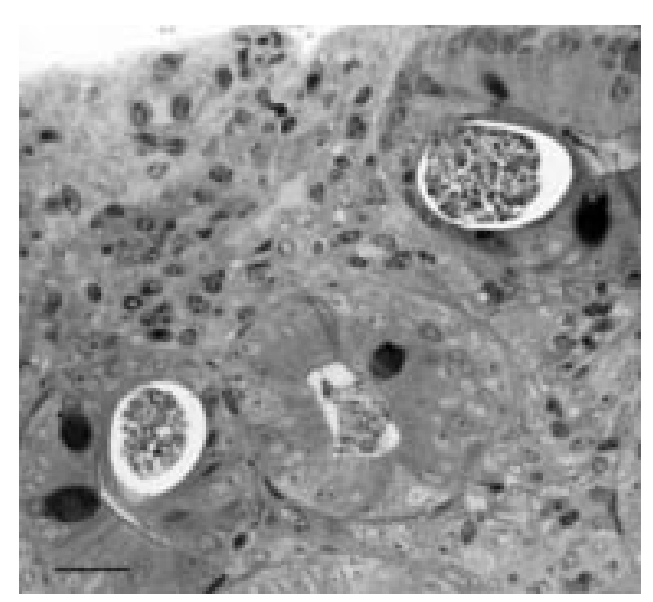

Figure 1 Morular eggs within crypts of Lieberkuhn. There is a sparse inflammatory cell infiltrate within the lamina propria. Bar $=19 \mu \mathrm{m}$. 


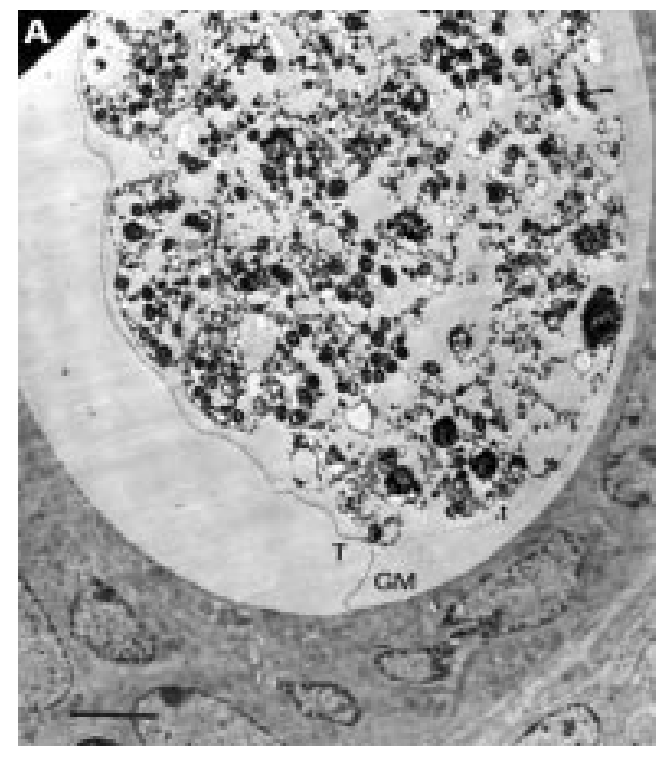

\section{Methods}

CASE HISTORY

A 33 year old HIV positive, drug misusing homosexual Brazilian man presented with a 20 day history of remittent fever, anorexia, abdominal pain with occasional vomiting, and a $5 \mathrm{~kg}$ weight loss. On examination he appeared slightly febrile and dehydrated and oral candidiasis was documented. There was diffuse abdominal tenderness, but bowel movements were normal. Moreover, an irregular mass in the rectum was revealed and both skin and oral mucosa lesions suggesting Kaposi's sarcoma were seen. Routine biochemical analysis showed no abnormalities but his CD4+ T cell count was $5 \times 10^{6} /$ litre. Stool examination

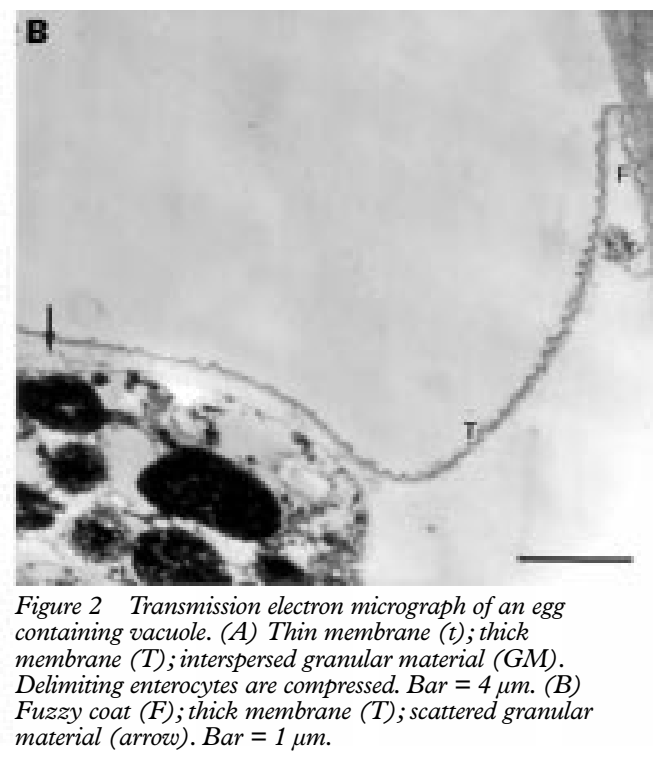

revealed rhabditoid larvae of $S$ stercoralis with negative results for bacteria, mycobacteria, Clostridium difficile toxin, mycetes, adenoviruses, rotaviruses, microsporidia, and other parasites. Computed tomographic scanning of the chest showed cavitations in the right upper lobe with diffuse miliary nodes, and tuberculous bacilli were grown in samples of bronchoalveolar lavage obtained at bronchoscopy. In addition, Kaposi's sarcoma was revealed both at bronchoscopy and in endoscopic biopsy specimens taken from the rectal mass.

The patient was diagnosed as having AIDS and belonging to the $\mathrm{C} 3$ stage, according to the 1993 CDC revised classification system.

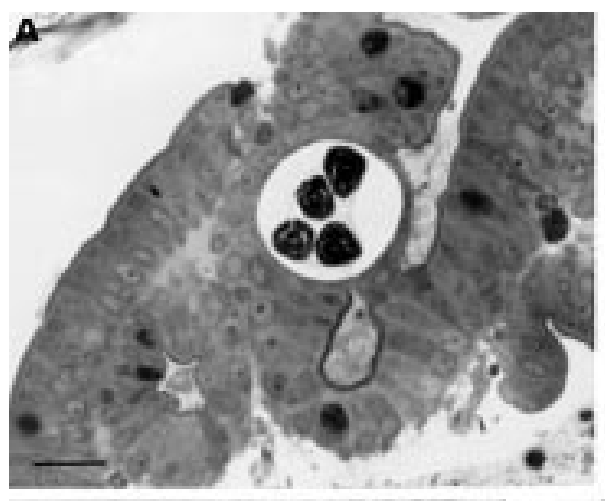

Figure 3 Newly hatched larvae within vacuole. $(A)$ A linear profile surrounds all larvae. Bar $=30 \mu \mathrm{m}$. (B) Transmission electron micrograph: thin membrane (t); thick membrane (T); fuzzy coat $(F)$. Space within thin membrane is clear. Bar $=2$ um. (C) Transmission electron micrograph: thin membrane ( $t$ ); fibrillogranular material (FGM). The nucleus of a delimiting enterocyte is elongated. $A$ small number of lipid droplets $(L)$ and scarce dense bodies (DB) are visible. Bar $=2 \mu \mathrm{m}$.
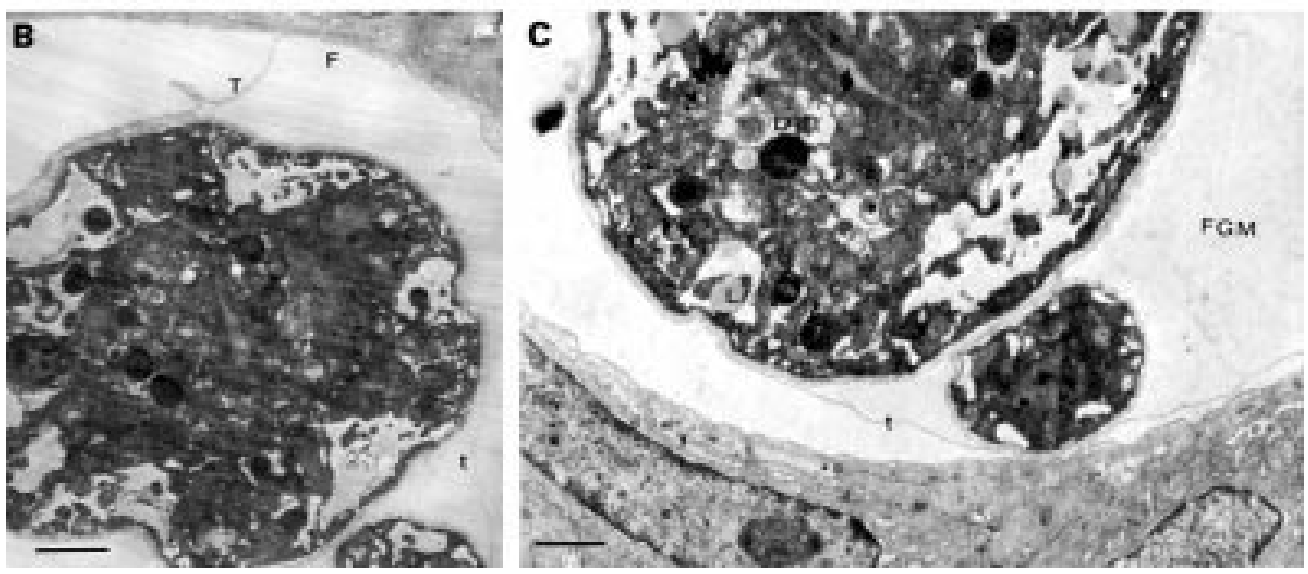

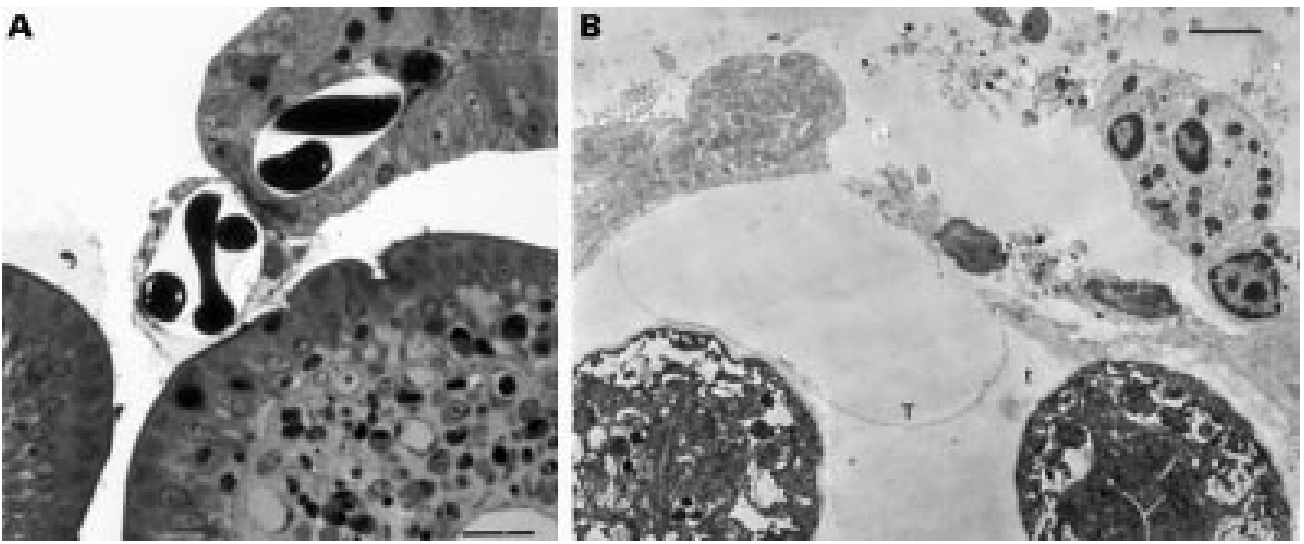

Figure 4 (A) Light microscopy. Release into the gut lumen of larvae still lying within vacuole. A linear structure surrounds all larvae. An inflammatory cell infiltrate within the lamina propria is visible. Bar $=30 \mu m$. (B) Transmission electron microscopy. Release into the gut lumen of two larvae lying within a broken vacuole. Thin membrane (t); thick membrane $(T)$. Bar $=3 \mu \mathrm{m}$.

Liver and spleen enlargement without focal lesions, and a peritoneal fluid collection, were also detected by computed tomographic scanning of the abdomen. Biopsy specimens were taken at endoscopy from the second portion of duodenum, and were processed for transmission electron microscopy (TEM). After endoscopy, antiparasitic treatment was started with oral albendazole $800 \mathrm{mg}$ daily for eight days, together with antituberculous drug treatment.

The patient rapidly improved and was asymptomatic at the end of albendazole treatment. At this time stools were collected (one sample each day for three consecutive three days), and yielded negative results for $S$ stercoralis. Consent for post-treatment endoscopy was denied. Subsequently the patient moved to another city and was lost to the follow up.

Informed consent was obtained from the patient, and human experimentation guidelines of the authors' institutions were followed in the conduct of the research.

\section{TRANSMISSION ELECTRON MICROSCOPY}

Four biopsy specimens taken from the second part of duodenum were processed. Specimens were fixed in cold $4 \%$ glutaraldehyde in $0.1 \mathrm{M}$ cacodylate buffer, $\mathrm{pH} 7.4$, at room temperature for four hours, washed in the same buffer, and postfixed in $1 \%$ osmium tetroxide in $0.1 \mathrm{M}$
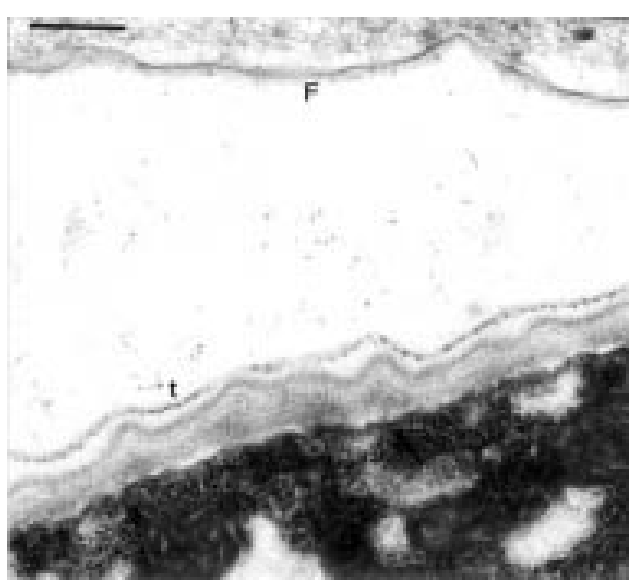

Figure 5 Transmission electron micrograph. Enterocyte and newly hatched larva forming opposing surfaces. Fuzzy coat $(F)$; thin membrane $(t)$. Bar $=3 \mu \mathrm{m}$. phosphate buffer, $\mathrm{pH} 7.4$, at $4^{\circ} \mathrm{C}$ for one hour. After dehydration through graded acetone series, the specimens were passed through propylene oxide and were then embedded in Epon 812. Semithin sections ( $3 \mu \mathrm{m})$ were cut, stained with toluidine blue sodium-tetraborate, and studied by light microscopy. About 100 ultrathin sections were obtained from all the biopsy specimens, were stained with uranyl acetate and alkaline bismuth subnitrate, and then examined by TEM. Care was taken to evaluate only areas away from the borders of the biopsy specimen to avoid traumatic artefact.

\section{Results}

At light microscopic examination, morular eggs measuring 40-50 $\times 24-30 \mu \mathrm{m}$ were found in the crypts of Lieberkuhn among the enterocytes (fig 1). Up to two eggs within each crypt were seen. The eggs were each lying in an apparent vacuole delimited by compressed and

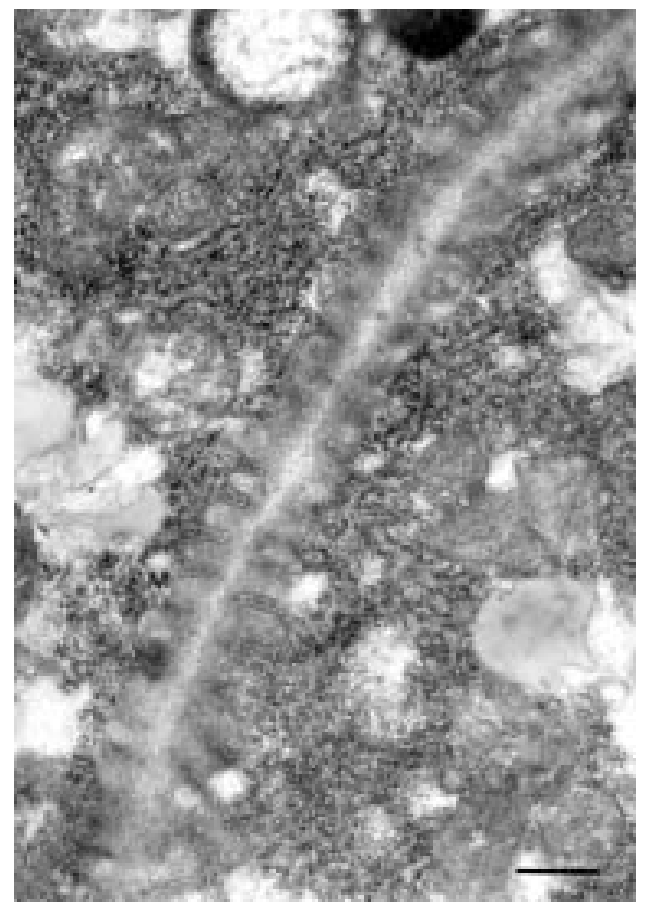

Figure 6 Transmission electron micrograph. Gut lumen. Microvilli (M). Bar $=0.2 \mu \mathrm{m}$. 


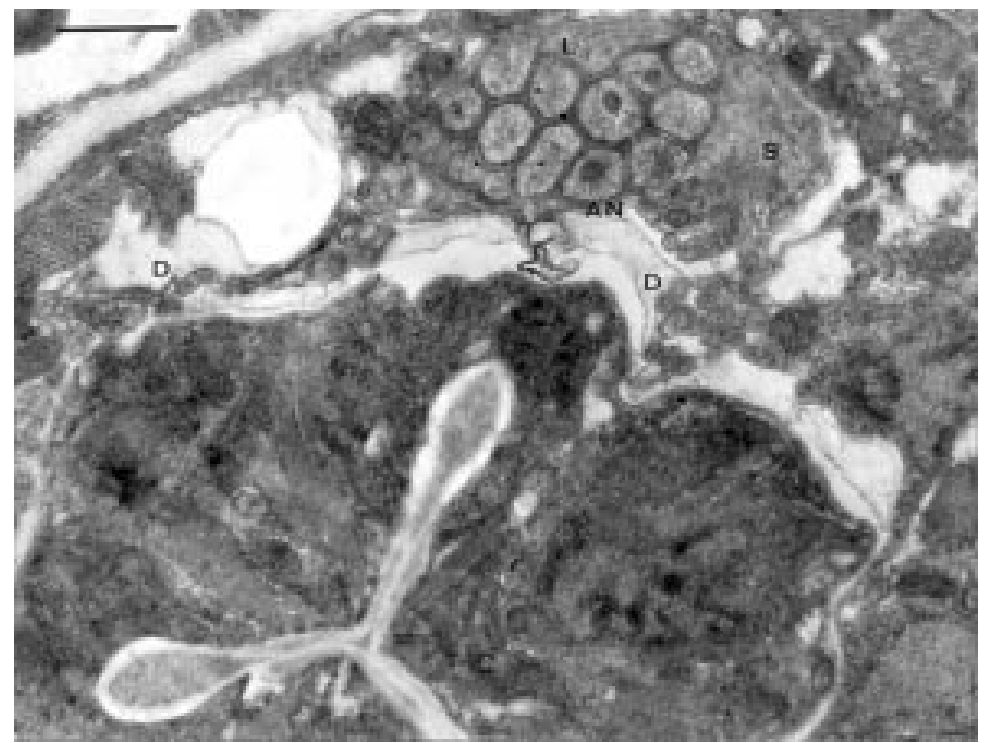

Figure 7 Transmission electron micrograph. Amphidial neurones $(A N)$; sheath cell $(S)$; lamellar cell (L). An almost closed gut lumen containing an amorphous material is seen. Dendritic processes medial to the amphidial neurones (D). Bar $=1 \mu \mathrm{m}$.

distorted enterocytes. On TEM examination, the nuclei of these enterocytes were elongated (fig 2) but no abnormalities of mitochondria or other subcellular organelles were found. A thin continuous monolaminar membrane surrounding the egg and a thick outer membrane, paralleling the thin membrane in some areas, were seen; these were separated by a space filled with scattered granular material (fig 2). A fuzzy coat on the surface of the enterocytes delimiting the vacuoles was also observed, showing that its detachment from the enterocytes accounted for the thick membrane and probably for the scattered granular material as well (fig 2).

Newly hatched larvae were observed by light microscopy and TEM of the semithin and ultrathin sections obtained (fig 3). They measured $12-20 \mu \mathrm{m}$ in diameter and were lying among the enterocytes within the same vacuole as the egg stage, although some larvae were free in the gut lumen. Up to four larvae were found within each vacuole, all surrounded by the same thin membrane that was present at the previous egg stage, as demonstrated by TEM (fig 3). The space within the thin membrane was clear although sometimes it contained scattered fibrillogranular material (fig 3). Release into the gut lumen of larvae still lying within vacuole was also observed by light microscopy and TEM (fig 4). Persistence of a fuzzy coat on the surface of the enterocytes delimiting the vacuoles (fig 5), and of a thick membrane (resulting from detachment of the fuzzy coat) partially surrounding the larvae and in some places paralleling the outside surface of the thin membrane, were both documented by TEM (figs 3 and 4). Light microscopy consistently showed a linear structure (probably resulting from the membranes described above) surrounding all larvae within each vacuole, whether they were within the gut mucosa or had just been released into the gut lumen (figs 3 and 4).

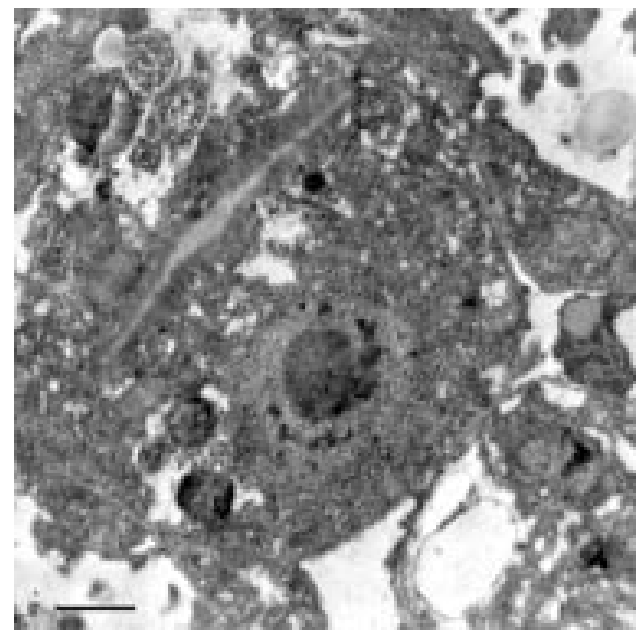

Figure 8 Transmission electron micrograph. Cell body of a neurone lacking nuclear membrane. Bar $=1 \mu \mathrm{m}$.

Only a sparse inflammatory infiltrate, mainly lymphocytes and plasmacytes, was noted in the lamina propria (figs 1 and 4).

Newly hatched larvae had a wavy $1.8 \mu \mathrm{m}$ thick cuticle which showed a dense surface monolayer overlying an amorphous less dense matrix exterior to the worm body (fig 5). These cuticular features were present in all the larvae studied, whether lying in the mucosa or just released into the gut lumen (figs 3 and 4).

The somatic cells of these larval stages were packed with ribosomes and elements of rough endoplasmic reticulum. A few lipid droplets and sparse dense bodies were also visible, but no Golgi bodies were seen in the ultrathin sections examined (fig 3). The brush border intestinal microvilli of larvae were slender, blunted, and regularly arranged, with no bulbous apical tips (fig 6). Pointed tips were rarely seen. Microvilli were included in a thick dense glycocalyx, the apical edge of which was not clearly delineated (fig 6). The gut lumen was usually closed and empty, although sometimes it contained amorphous material (figs 6 and 7).

Both chemoreceptor amphidial neurones and other neurones were evidenced by TEM at various levels along the larval body. All of the 13 dendritic processes of the amphidial neurones were seen within the channel formed by the sheath cell (a goblet shaped supporting cell), and showed tight interdendritic junctions and very few lamellae of the specialised "lamellar cell" neurone (fig 7).

Dendritic processes of other, probably mechanosensory, neurones were also seen in distinct bundles medial to the amphidial channel (fig 7). Cell bodies of neurones lacking a nuclear membrane were found (fig 8), as were three complexely arranged dendritic processes. The first dendrite showed an electron-dense thick ring which paralleled a less dense inner ring surrounding three osmiophilic bodies (fig 9); the second showed two electron-dense irregular concentric rings containing a densely stained central body (fig 9); the third appeared as an electron-dense irregularly round shaped structure containing an osmiophilic central body surrounded by an electron-dense ring (fig 9). 

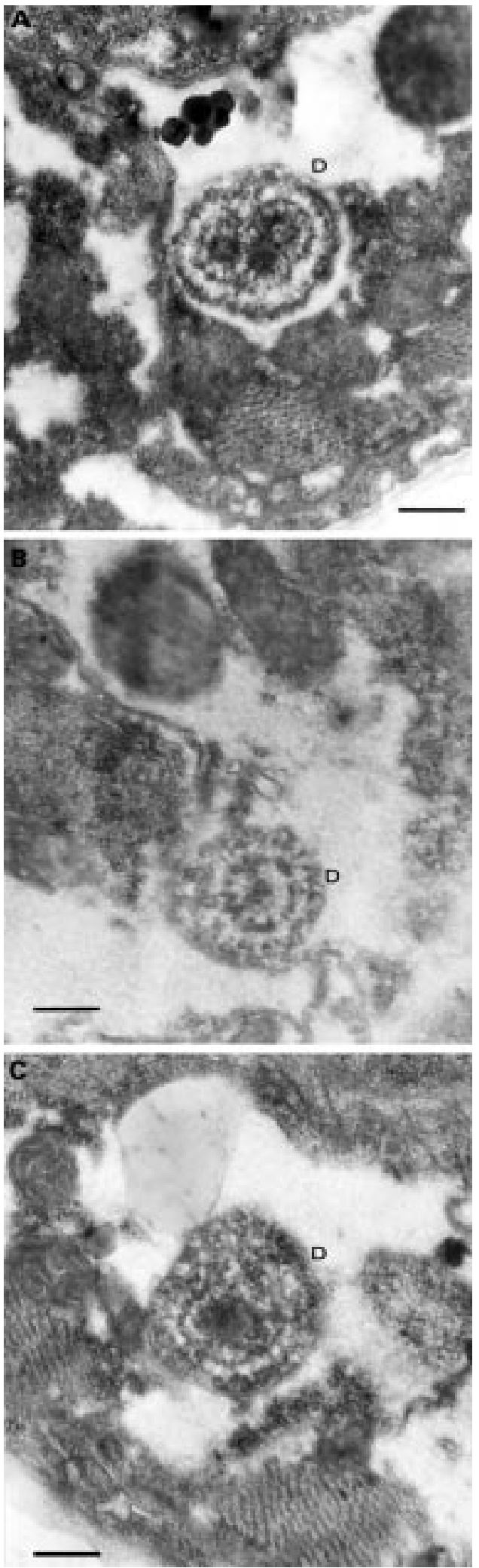

Figure 9 Transmission electron micrograph. $(A),(B)$, (C): dendritic processes (D). Bars $=0.4,0.2$, and $0.3 \mu \mathrm{m}$, respectively.

Segmentary spike-like waves, caused by contractures of worm body musculature, were sometimes present on the surface of newly hatched larvae, suggesting active movements (fig 10).

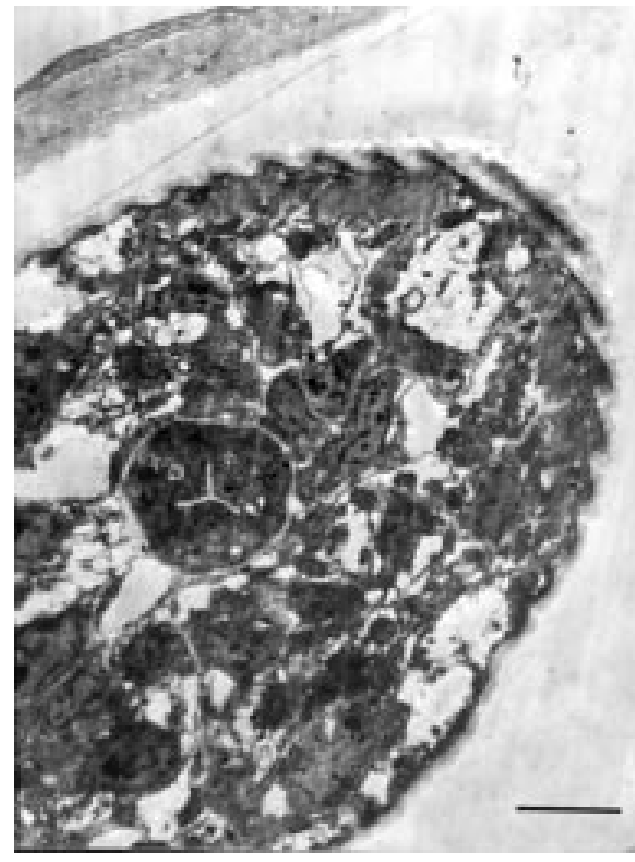

Figure 10 Transmission electron micrograph. Spike-like waves. The thin membrane is visible. Bar $=2 \mu \mathrm{m}$.

\section{Discussion}

To date, no studies on newly hatched larvae of Strongyloides stercoralis, either in animals or in humans, have been published. Furthermore, there are no published reports concerning the ultrastructural features of this nematode in the human intestinal tract.

To our knowledge, the present study is the first describing newly hatched larvae within small intestinal mucosa and it is also the first ultrastructural study of $S$ stercoralis in the human.

The data provided by examination of the semithin sections confirm that the crypts of Lieberkuhn are the site of egg deposition by parthenogenic females in the human intestine. The results obtained by TEM examination also enabled us to conclude that the apparent parasitophorous egg containing vacuoles probably represented cross sections of the tunnels resulting from the migration of adult females through the epithelium. Indeed, the ultrastructure of these vacuoles was almost identical to that of vacuoles harbouring the adult female form of $S$ stercoralis which were described in the jejunum of immunosuppressed dog as cross sections of the tunnels formed by worm movements. ${ }^{6}$ Being the natural milieu for adult females, tunnels are the expected site of egg deposition, as was shown by the results of a TEM study on the location of Strongyloides ratti in the mouse intestine. ${ }^{9}$

Both the active movements suggested by TEM in newly hatched larvae and their persistence in an apparent parasitophorous vacuole until they were released into the gut lumen are in agreement with the above considerations. Indeed, these findings suggest that newly hatched larvae are able to break junctional complexes between adjoining enterocytes and to move actively between them, so prolonging the tunnel where they were born 
until they reach the intestinal lumen. Mechanical damage caused by the resulting compression was shown by light microscopy and TEM in the enterocytes bordering the vacuoles.

The nature of the fuzzy coat seen on the surface of enterocytes opposing either the eggs or the newly hatched larvae is unknown. It could be derived from host, helminth, or both, and might be formed from the remnants of necrosed cells and antigen-antibody complexes. Detachment of the fuzzy coat from the enterocytes accounted for the thick membrane that persisted around both eggs and newly hatched larvae. The possibility that detachment was an artefact is unlikely as it was observed in several larval and egg stages. We therefore assume that the thick membrane could represent a protective tegument for the parasite during its development within the small intestinal mucosa. The same function is conceivable for the continuous monolaminar thin membrane which was seen to surround developing parasites from the egg to the larval stage. Persistence of both these membranes until the larvae were released into the gut lumen, as evidenced by TEM, is an interesting finding that could throw some light on the mechanisms involved in protecting immature forms of $S$ stercoralis against host defences. Although either a parasitic or a host source for these membranes is conceivable, TEM evidence supported a parasitic origin for the thin membrane, and strongly suggested a host origin for the thick membrane.

As newly hatched larvae were located in the gut mucosa among the enterocytes, they were open to attack by inflammatory cells. ${ }^{13-15}$ However, probably as a result of advanced immunodeficiency of the host, only a sparse inflammatory infiltrate was noted in all the semithin sections studied, ${ }^{6}$ and the body cells of these larval stages showed no signs of ultrastructural damage (as shown by the presence of only scanty lipid droplets and dense bodies and by the ample presence of ribosomes and rough endoplasmic reticulum). ${ }^{11}{ }^{12}$

Newly hatched larvae were probably a non-feeding or a poorly feeding stage, as their intestinal lumen was closed and empty (although it sometimes contained amorphous material), and no buds were seen on the microvilli. ${ }^{11}{ }^{12}$ Published reports show that these buds-which form at the tips of the microvilli in feeding worms of many genera and species as a probable mechanism for secreting enzymes into the gut lumen or for shedding older membrane proteins-are lost in starved or immune damaged worms, and such worms also show a scarcity or absence of food in their intestinal lumen. ${ }^{516} 17$ Therefore, the absence of buds observed at the tips of the microvilli in newly hatched larvae may provide further support for the view that these represent a non-feeding or a poorly feeding stage. This could indicate immaturity of the main chemoreceptors, the amphidial neurones, which are known to be directly involved in parasite feeding through their sensitivity to osmotic stimuli from the host. ${ }^{3}$ Indeed, the almost complete absence of lamellae of the amphidial lamellar cell in newly hatched larvae, as compared with their presence in free living larvae, ${ }^{3}$ seems consistent with this hypothesis. On the other hand, no immaturity of the mechanosensory neurones, which play a role in migration of larvae, ${ }^{4}$ was be expected as many larvae were seen to be released into the gut lumen, thus suggesting an ability to move through the epithelium. In agreement with this, no ultrastructural evidence of immaturity was found either in the complexely arranged dendrites (fig 9) or in those medial to the amphidial channel (fig 7), all of which showed similarities to the mechanosensory dendrites described in free living third stage larvae. ${ }^{4}$

There were no differences in thickness between the cuticles of newly hatched larvae and those of adult worms, as judged by comparison with parthenogenic females of $S$ stercoralis lying in the jejunum of the immunosuppressed dog. ${ }^{6}$ Marked cuticle immaturity was documented, however, in newly hatched larvae. Indeed, cuticular features typical of more mature larvae and adult worms of Strongyloides species (such as annulations, grooves, the three layer epicuticle, and the deeper amorphous, striated, and basal layers) were all absent in newly hatched larvae. ${ }^{6-8}$ Nothing is known about the role this immaturity plays in the equilibrium between the harmful potential effects of the worm and the defences of the host. It is probable, however, that the immature cuticle found in newly hatched larvae may be of some disadvantage to the parasite. These considerations are in agreement with the protective role suggested for both the thin and the thick membranes which were found to surround the worms from their egg stage to the release of larvae into the gut lumen. The need for protection could be even more necessary during this period considering that no cuticular maturation was evidenced on TEM at the time of release of the larvae into the gut lumen.

The thin and thick membranes may also provide a morphological explanation for a phenomenon observed in Strongyloides ratti in which radionuclide and TEM evidence suggests that infective larvae lose a surface coat when they penetrate the skin. ${ }^{78}$ This coat could well be either the thin or the thick membrane described by us, if one assumes that it is not lost when rhabditiform larvae develop into infective larvae.

\section{CONCLUSIONS}

Ultrastructural data from this study indicate that newly hatched larvae of $S$ stercoralis are an immature stage. Our results throw some light on the earliest phases of the life cycle of $S$ stercoralis within small intestinal mucosa, and on the natural history of this parasitosis in the human. The results reported here, however, were obtained from a patient with high grade cellular immunodeficiency and it is possible that the findings would be different in an immunocompetent host. Therefore, TEM studies in immunocompetent hosts are required to elucidate the natural history of this parasitosis further in the human. 
We thank Enrico Tortoli (Laboratory for Microbiology, Careggi Hospital, Florence) for critical review of the manuscript.

1 Schad GA. Morphology and life history of Strongyloides stercoralis. In: Grove DI. Strongyloidiasis: a major round-
worm infection of man. London: Taylor and Francis, worm infection

2 Mahmoud AAF. Strongyloidiasis. Clin Infect Dis 1996;23 949-53.

3 Ashton FT, Bhopale VM, Fine AE, et al. Sensory neuroanatomy of a skin-penetrating nematode parasite: Strongyloides stercoralis. I. Amphidial neurons. F Comp Neurol 1995;357:281-95.

4 Fine AE, Ashton FT, Bhopale VM, et al. Sensory neuroanatomy of a skin-penetrating nematode parasite Strongyloides stercoralis. II. Labial and cephalic neurons. $\mathcal{F}$ Comp Neurol 1997;389:212-23.

5 Schad GA, Thompson F, Talham G, et al. Barren female Strongyloides stercoralis from occult chronic infections are rejuvenated by transfer to parasite-naive recipient hosts and give rise to an autoinfective burst. $\mathcal{F}$ Parasitol 1997;83:78591 .

6 Grove DI, Warton A, Yu LL, et al. Light and electron microscopical studies of the location of Strongyloides stercoralis in the jejunum of the immunosuppressed dog. Int f Parasitol 1987;17:1257-65.

7 Grove DI, Warton A, Northern C, et al. Electron microscopical studies of Strongyloides ratti infective larvae: loss of the surface coat during skin penetration. F Parasitol 1987;73:1030-4

8 Martinez AM, De Souza W. A freeze-fracture and deep-etch study of the cuticle and hypodermis of infective larvae of Strongyloides venezuelensis (Nematoda). Int $\mathcal{f}$ Parasitol 1997;27:289-97.
9 Dawkins HJ, Robertson TA, Papadimitriou JM, et al. Light and electron microscopical studies of the location of Strongyloides rat

10 Zaman V, Dawkins HJ, Grove DI. Scanning electron microscopy of the penetration of newborn mouse skin by Strongyloides ratti and Ancylostoma caninum larvae. Southeast Asian f Trop Med Public Health 1980;11:212-19.

11 Moqbel R, McLaren DJ. Strongyloides ratti: structural and functional characteristics of normal and immune-damaged worms. Exp Parasitol 1980;49:139-52.

12 Moqbel R, McLaren DJ, Wakelin D. Strongyloides ratti: reversibility of immune damage to adult worms. Exp Parasitol 1980;49:153-66.

13 Neva FA. Biology and immunology of human strongyloidiasis. F Infect Dis 1986;153:397-406.

14 Kerlin RL, Nolan TJ, Schad GA. Strongyloides stercoralis: histopathology of uncomplicated and hyperinfective histopathology of uncomplicated and hyperinfective strongyloidiasis in the mongolian gerbil, a rodent model
human strongyloidiasis. Int f Parasitol 1995;25:411-20.

15 Trajman A, MacDonald TT, Elia CCS. Intestinal immune cells in Strongyloides stercoralis infection. 7 Clin Pathol 1997;50:91-5.

16 Bonner TP. Changes in the structure of Nippostrongylus brasiliensis intestinal cells during development from the free-living to the parasitic stages. F Parasitol 1979;65:74550.

17 Misch DV. Intestinal microvilli: responses to feeding and fasting. Eur f Cell Biol 1980;21:269-79.

18 Grove DI, Northern C, Warwick A, et al. Loss of surface coat by Strongyloides ratti infective larvae during skin penetration: evidence using larvae radiolabelled with ${ }^{67} \mathrm{gal}-$ lium. F Parasitol 1984;70:689-93. 